\title{
Tecnología para la reforestación en América Latina
}

\author{
Michael R. Keyes Hennin ${ }^{1}$
}

\begin{abstract}
RESUMEN
La deforestación ha tomado ventaja inicial frente a los esfuerzos aplicados a la reforestación. Existen antecedentes históricos en algunas naciones acerca de las consecuencias de la ambición por colonizar y desarrollar actividades en la frontera agrícola. Ello provoca la disminución de la superficie forestal. El presente trabajo tiene como objetivo ver las semejanzas entre los programas históricos y los actuales relativos a la conservación y la restauración de los suelos forestales degradados por la agricultura, y sobre todo, a la tecnología desarrollada para apoyar la reforestación enfatizando casos de América Latina.
\end{abstract}

\section{PALABRAS CLAVE}

Deforestación, reforestación, conservación de suelos, trasplantes mecanizados

\section{INTRODUCCION}

La deforestación se debe entender no sólo como resultado de la acción evolutiva del hombre individual, sino como elemento de las relaciones dinámicas entre las culturas, sus respectivas tecnologías y las características del medio físico. Uno de los retos para los profesionistas forestales

\begin{abstract}
Deforestation has surpased reforestation efforts throughout the tropics. There are a plethora of historical precedents for the consequences of ambitious nations with frontier style colonizing and agricultural development which has led to a reduction of forested areas. The objectives of this paper are to review the similarities between the development of conservation and restoration programs for forest soils degraded by agriculture and, above all, the technology developed to aid reforestation, emphasizing efforts in Latin America.
\end{abstract}

\section{KEY WORDS}

Deforestation, reforestation, soils conservation, mechanized planting

hoy en día, es el entender las interrelaciones entre las diversas acciones en el tiempo y en el espacio para optimizar los esfuerzos de las diversas acciones dedicadas a la reforestación. En el presente, igual que ha sucedido en cada época de la humanidad, existen grupos sociales dominantes que cuentan con la tecnología, y dirigen la explotación de los

1 Investigador. Departamento de Productos Forestales y Conservación de Bosques. Instituto de Ecología, A.C. Apdo Postal 63. 91000 Xalapa, Veracruz, México.

Manuscrito recibido para su publicación el 26 de Septiembre de 1995 
recursos naturales, implicando a grupos humanos autóctonos. Históricamente, los casos más exitosos han sido aquellos en que se aprovecha lo mejor de la tecnología.

El presente trabajo tiene como objetivo ver las semejanzas entre los programas históricos y los actuales, relativos a la conservación y a la restauración de los suelos forestales degradados por la agricultura, y sobre todo a la tecnología desarrollada para apoyar la reforestación.

\section{CAUSAS DE LA DEFORESTACION}

La colonización del planeta por humanos, así como el uso de herramientas y del fuego, empezaron a cambiar la vegetación de algunos ecosistemas. Por ejemplo, los aborígenes de Australia propiciaron que la vegetación se convirtiera en pirófila y pirotolerante. Posteriormente, la intensidad de incendios antropogénicos aumentó en perjuicio de los bosques, y en la merma de árboles en las praderas (Schule, 1990), sostiene, además que durante este proceso de aprendizaje y durante los milenios de uso del fuego sin cuidado suficiente, se ha sometido al continente africano a un proceso de sabanización progresiva y de desertificación.

Como se puede inferir, las acciones humanas han estado estrechamente relacionadas con la vegetación desde mucho tiempo antes del auge de las grandes civilizaciones. El progreso de éstas empezó a poner en entredicho todo el balance entre el usuario y el recurso forestal en algunas partes del mundo. Desde los tiempos preagrícolas se estima que los bosques del mundo se han reducido entre cinco y seis mil millones de hectáreas; el más alto porcentaje (32-35\%) corresponde a bosques templados (WRI, 1990).
Entre los casos más dramáticos de deforestación se encuentra el de América Latina, que asciende a aproximadamente 12.2 millones de hectáreas por año (WRI, 1990). Desafortunadamente, no existen cifras confiables que reflejen la proporción relativa de deforestación causada por los desmontes en la agricultura de subsistencia como tampoco hay para el establecimiento y la ampliación de potreros, la agricultura trashumante, las actividades de extracción maderables y no maderables. Lo cierto es que una gran proporción de la deforestación de 890,000 hectáreas en Brasil y 340,000 hectáreas de bosques perdidos en México, año con año, tiene que ver ahora con los programas de colonización en zonas selváticas. Los incrementos de población humana en las selvas están ligados también a la penetración de los mercados internacionales relativa a los productos tropicales como tabaco, carnes rojas, frutos y aceites de las palmas y cítricos. La conversión de la cobertura arbórea natural en plantaciones para los cultivos tropicales perennes como hule, plátano, quina, caña, mango, etc., ha sido ampliamente documentada (Hamilton,1990; Honadle, 1992). Además de lo agroindustrial, nuevos esquemas han surgido recientemente: tal es el caso de las "narcoreservas" donde aparentemente un grupo de "biólogos" laboran en áreas limitadas, mientras los "guardabosques" cumplen con el doble propósito de limitar el acceso y apoyar en las actividades del negocio en el sitio, representando una fuerte competencia para el recurso forestal por la relación costo-área-tiempo.

\section{LA FRONTERA Y LA NUEVA FRONTERA AGROPECUARIAS}

En realidad las presiones sobre los bosques y las selvas dejan pocas posibilidades para que la regeneración natural se establezca. Si ello sucediera 
sería mucho esperar que se caracterizara como una vegetación comercial, cuando ni siquiera la vegetación primaria del sitio sería lo suficientemente rica en etnoflora (flora útil al hombre o a la comunidad cercana) como para poder evitarse más desmontes a futuro. Visto desde otra óptica, sabemos que en la ampliación de la frontera agrícola se conjugan múltiples factores interrelacionados con el deterioro del bosque pero que también podrían ser modificados para la conservación.

Los numerosos ejemplos de actividades localizadas en las fronteras agrícolas pueden enseñar mucho, dado que en los últimos siglos las culturas occidentales han cometido en repetidas ocasiones un sinnúmero de errores y han encontrado un número similar de remedios en las respectivas fases de colonización y reforestación. Por "frontera", a juicio del autor, se debe entender el conjunto de límites de la civilización dominante u operante, mintras que por "nueva frontera agropecuaria", se entiende el conjunto de actividades actualmente consideradas como "productivas" tanto agrícolas como pecuarias, independientes o escalonadas en tiempo.

\section{LA TECNOLOGIA PARA LA REFORESTACION}

Ya han pasaron más de dos décadas desde que se inició el impulso al uso de tecnología por los sectores oficiales forestales a nivel mundial. Sin embargo, no fue considerado el uso de maquinaria para la reforestación masiva como parte integral de las operaciones forestales sino hasta hace relativamente poco tiempo (FAO, 1983). Poco ha sido escrito sobre el aspecto tecnológico de las fronteras. La tecnología empleada en la frontera angloamericana fue una consecuencia lógica para ahorrar en tiempo y para optimizar el empleo de mano de obra y alcanzar en forma casi individual la escala de reforestación que las condiciones pidieron en cada comunidad.

Hay que destacar que en el caso de EUA, entre los años veinte y treinta, no fue casual el hecho de que la maquinaria para el uso forestal proviniera de la maquinaria agrícola. Los granjeros descubrieron el alcance de la mecanización y utilizaron sus tractores con arados modificados para abrir y cerrar surcos. Por otra parte, la sequía de los años treinta forzaba a los trabajadores del campo, por necesidad de sobrevivencia, a buscar opciones complementarias para proteger el suelo mediante cortinas rompevientos y así asegurar algún porciento de la producción.

Auxiliados por técnicos en la conservación y obligados por ley, la tecnología impulsó uno de los esquemas más grandes de reforestación atestiguados en el continente americano. Durante este período de colonización en EUA, el desarrollo de la mecanización agrícola apoyó a los granjeros para alcanzar mejores logros, tanto en la producción como en la reforestación. Lo más relevante desde el punto de vista silvícola tiene que ver con lo último. Es necesario poner énfasis en que fueron los mismos trabajadores del campo quienes lograron alcanzar aquel cometido.

Para alcanzar el logro de esos objetivos de reforestación contaron con elementos no muy distintos de los existentes ahora. Hubo el apoyo por parte del estado y también ocurrió durante otra de las épocas de degradación ambiental acelerada. La innovación fue una parte esencial para optimizar el desempeño de una amplia variedad de tareas en la reforestación en armonía con la experiencia y el nivel técnico que poseían aquellos abocados a 
la reforestación. Por ende, llegaron a manejar métodos múltiples para alcanzar la diversidad de metas y para adaptarse a las condiciones locales de tecnología con que contaban.

Conceptualmente, los diseños originales provinieron de maquinaria de trasplante de viveros hortícolas, cultivos frutícolas y de hortalizas (Lawyer, 1974). Visto desde el punto de vista histórico, el desarrollo de la maquinaria para trasplantadoras ha cumplido ya más de 100 años, desde que fue concebida la primera patente (Op. cit.). En todos los casos, esas técnicas fueron adaptaciones de herramientas agrícolas. Inicialmente, la tracción animal fue probada y utilizada en la práctica a partir de los años veinte y treinta. Paulatinamente, el uso de animales dio lugar a maquinaria de arrastre de llanta $u$ oruga. En la etapa actual del proceso de evolución de la tecnología para la reforestación, ahora se cuenta con maquinaria motorizada independiente, cuyo costo mínimo es de alrededor de $\$ 27,000$ dólares (McKenzie et al., 1984). No obstante, la maquinaria actual para la industria forestal comercial opera básicamente sobre los mismos principios con los que fue desarrollada en los años cuarenta. La tecnología para plantaciones comerciales actualmente utilizada, ha sido poco perfeccionada desde finales de la década de los cincuenta. En forma sencilla, estos tipos de maquinaria utilizados en reforestación se clasifican como sigue: máquinas de surco continuo, máquinas intermitentes y sembradoras (Noecker, 1967; Steinbeck y McAlpine, 1973).

El surco continuo ha sido empleado para arar desde los inicios de la agricultura en Egipto. Desde luego, los animales de tiro fueron y siguen siendo utilizados en las labores del surcado. La figura 1 muestra la adaptación del arado estilo "chiapaneco" para abrir surcos continuos ( $y$ a veces intermitentes) para la reforestación, posteriormente, se procede a colocar la plántula en forma manual y se cierra el surco alrededor de la planta "tapa pie".

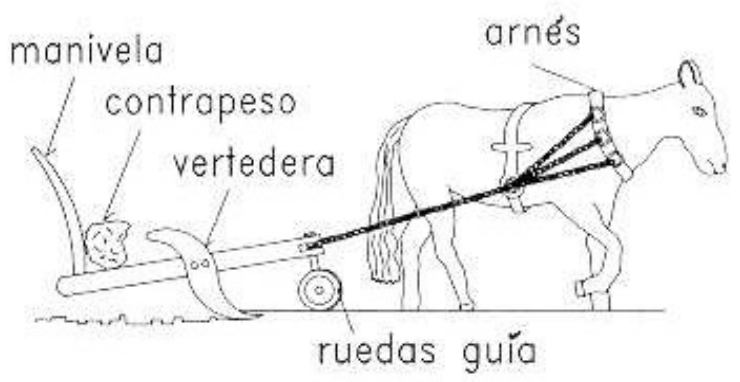

Figura 1. Arado "chiapaneco" usado en prácticas de reforestación

A pesar de que la mecanización en operaciones forestales ha cobrado mucha importancia, sobre todo en los países donde la mano de obra es cara, el uso de bestias lejos de reducirse, ha aumentado. Por ejemplo, el uso de caballos para el arrastre de trocería en California (EUA) no ha dejado de existir y está aumentando desde hace más de 20 años por ser más compatible con los intereses de conservar suelos en pendientes pronunciadas (Bamford y Bamford, 1981). Esta tecnología bien podría ser idónea en la mayor parte de los países en vías de desarrollo si algunos incentivos fueran implantados.

De hecho, existen muchas maneras para abrir un surco. En el Valle de México se ha usado maquinaria pesada para formar terrazas en el subsuelo sobre pendientes totalmente erosionadas, tras siglos de uso agrícola. El surco hecho por uno o dos timones para el "subsoleo" 
(Fig. 2) se aprovecha para la colocación de plántulas de envases y se tapa la cepa con pala. La reforestación mecanizada en laderas, ha sido exitosa en pendientes de hasta aproximadamente $38 \%$ dentro del programa gubernamental a cargo de la Comisión del Lago de Texcoco (Pimentel, 1995).

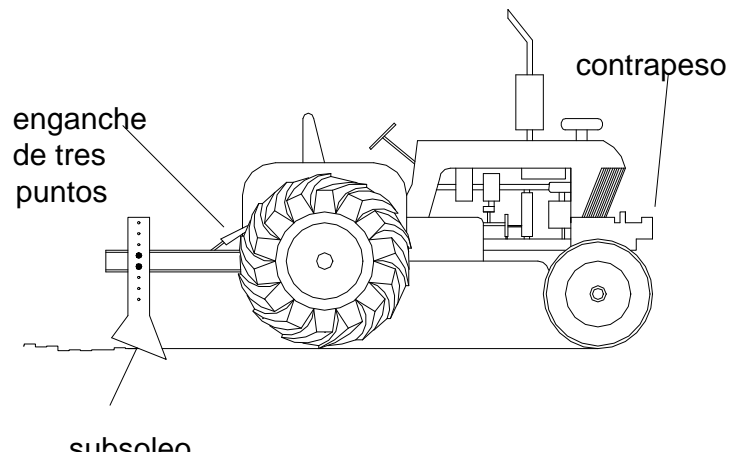

Figura 2. Tractor de oruga con subsoleo

Una de las máquinas trasplantadoras básicas más utilizadas ha sido aquella que está provista con un disco circular o "coulter" (estilizado en la figura 3) para hacer un corte continuo de suelo y raíces. Las configuraciones varían mucho. En este esquema se incluye un raspador de césped que normalmente es necesario en reforestación de potreros. Se puede montar una pala frontal con dientes sobre el tractor para que cumpla con esta última función. Casi siempre se incluye un par de "zapatos" (punzones) para abrir el surco cortado (que no aparecen en la figura 3). Inmediatamente detrás del espacio en donde se sienta una persona para colocar la planta se encuentra un par de ruedas remolcadas para aporcar el suelo sobre las raíces.

El surco continuo es muy operativo ya sea a través del disco circular o del arado modificado. La conceptualización de estos diseños iniciales proviene de maquinaria trasplantadora (para trabajos en horticultura, frutales pequeños $u$ hortalizas). Se capta lluvia sobre cada curva de nivel, mismo que es de gran beneficio en zonas áridas o semiáridas.

En zonas con precipitación pluvial mayor de $1,100 \mathrm{~mm}$ por año, se recomienda que las curvas de nivel tengan pequeñas desviaciones, para permitir el desagüe el surco, evitando así, que la humedad se concentre pudriendo a las raíces. Es laborioso tratar de hacerlo sin haber visto resultados primero. $\mathrm{La}$ sugerencia es que, las organizaciones busquen la manera de conseguir uno y luego vean la manera de reproducirlo y hacerle las modificaciones necesarias con base en materiales y necesidades locales. En general, la maquinaria de surco continuo ha sido y es, actualmente, la tecnología dominante para plántulas en reforestación comercial (Bagley, 1973; Hensel, 1958, 1959a, 1959b, 1959c, 1961; Hill, 1964; Holekamp, 1958). Esta misma puede ser impulsada por tractores convencionales o de oruga.

Las máquinas de surco intermitente pueden ser el recurso más simple para adecuar tractores que se tengan a mano para la reforestación semimecanizada. Para hacer las adaptaciones indicadas en la figura 4 , no se requiere mucha práctica, como es el caso de las máquinas tras plantadoras de surco continuo. Los modelos más sencillos se atornillan con las tuercas del "rin" trasero del tractor convencional, con una o dos varillas de metal. Estos pueden ser de una o de dos 


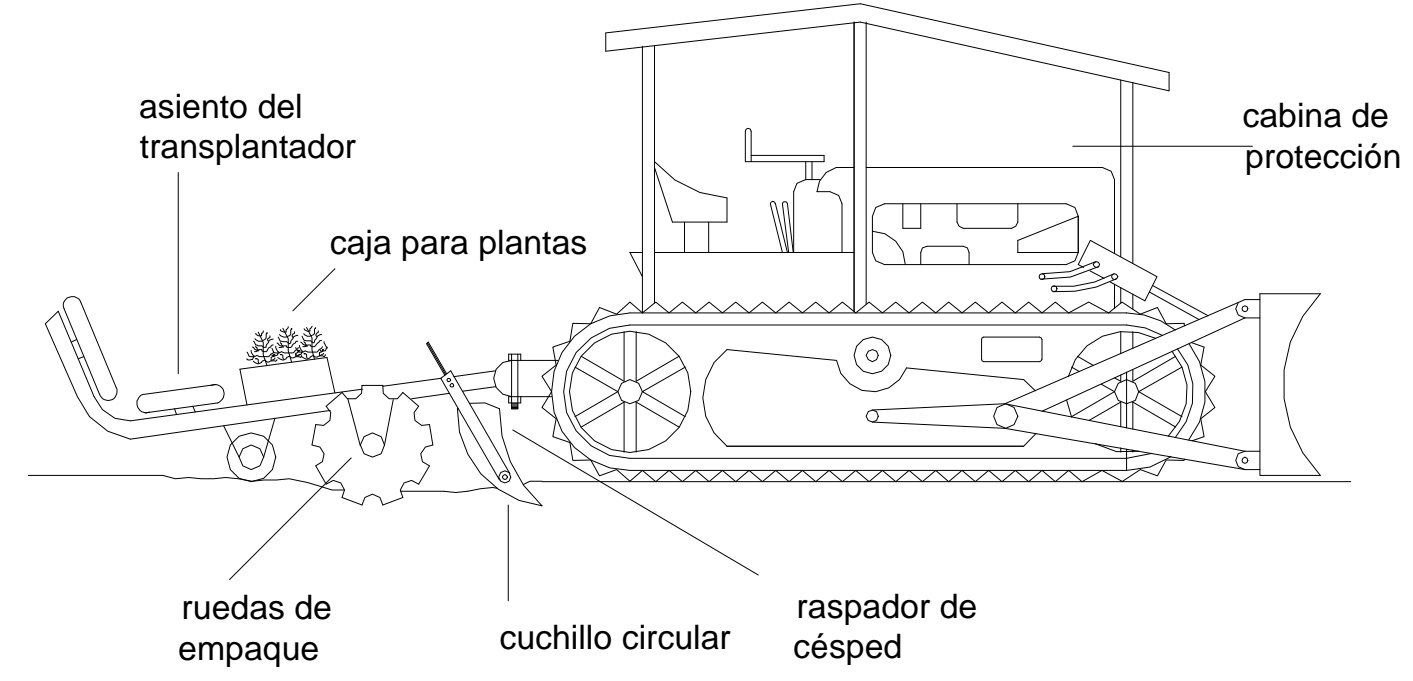

Figura 3. Máquina trasplantadora con "coulter" estilizado

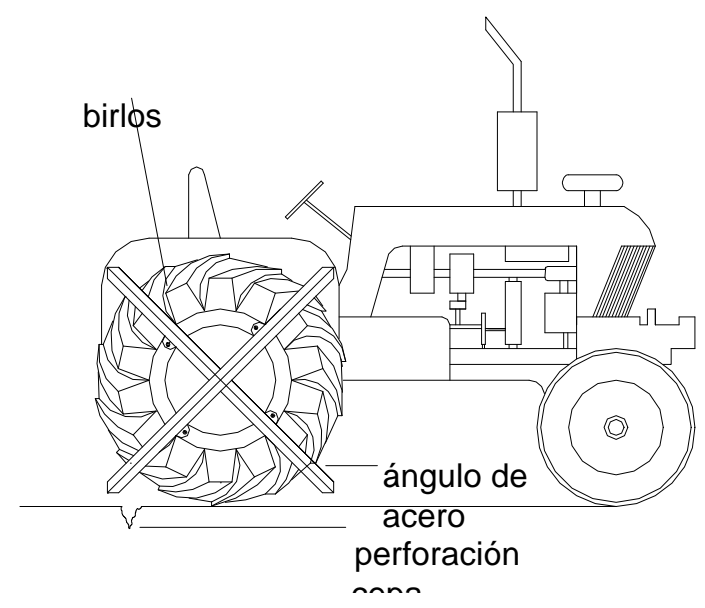

Figura 4. Adaptaciones para el tractor convencional de cava-hoyos ruedas. Al avanzar el tractor, la(s) varilla(s) sirve( $n)$ como cava-hoyos. El distanciamiento entre hoyos será entonces fijo, dependiendo del diámetro de la llanta y de la distancia entre las varillas; la profundidad será proporcional a la longitud de la punta de la varilla que servirá de punzón. Si está bien trabajado, el mismo sistema puede proporcionar tracción adicional en áreas resbalosas. No obstante lo anterior, con pendientes fuertes sobre tramos quebrados y en donde hay mucha roca, el alzamiento de la rueda trasera al alcanzar una piedra en "lomillas" puede representar un peligro para el operador, por lo que no se recomienda el uso de este sistema intermitente durante períodos de lluvia excesiva. Normalmente, no todos los hoyos resultan suficientemente limpios para la planta y debe haber un excedente de aquellos para escoger, además de que se requiere una varilla o pala pequeña a la mano a la hora de efectuar el trasplante. Rehacer los hoyos es algo importante, dado que no siempre es sencillo agregar la tierra alrededor de las raíces. 
Otra opción ya probada por el autor en conjunto con un alumno (Anduaga, 1988) en Tabasco, México consiste en modificar una reja normal o arado de doble vertedera soldando placas metálicas a las "alas" o la barra central. Después de efectuar la modificación, se montaron una o dos rejas sobre la barra portadora de los implementos. Con esa configuración el operador activa periódicamente este portaimplementos con el sistema hidráulico común y los resultados han sido muy favorables en el Estado de Tabasco, México. Es prudente hacer la observación que sin modificación alguna se pueden hacer hoyos con el sistema hidráulico del tractor. La ventaja del rellenado es que la mayor parte del suelo cae frente a cada cepa en lugar de caer en el interior de la misma conforme el tractor avanza.

Otras máquinas de surco (hoyo) intermitente han sido probadas y según McKnight (1976), a pesar de que los modelos son ya antiguos, una vez superados los problemas mecánicos, ofrecen ventajas en la operación y son comparables a la maquinaria de surco continuo. Una variante del surco intermitente es la inyección de plantas, ilustrada en la figura 5 (modificación de Altman, 1969). Este tipo de tecnología es menos estable y no tan globalmente utilizada (Stevenson, 1966); peor aún, está económicamente fuera del alcance del campesino común. No obstante, estas tecnologías estaban disponibles ya a nivel comercial hace bastante tiempo (USFS, 1967).

Las sembradoras manuales y semimecánicas son menos comunes en reforestación. Algunos tipos de sembradoras completamente mecánicas han sido utilizadas en tierras desprovistas de vegetación arbórea desde principios de siglo. Se hace enfasis que, en la siembra directa para reforestación, la mecanización no es tan necesaria como los cuidados de las plántulas después de sembrada. La siembra directa de semillas se puede hacer simultáneamente o poco después de la siembra de cultivos en la milpa. Conjuntamente, los cultivos y árboles (sistemas agroforestales) deben gozar de más cuidados que los árboles solos. Debe intentarse la siembra mecanizada únicamente en condiciones de poca competencia y siempre con buenas prácticas de cultivo como son las limpiezas frecuentes.

Como en otras técnicas de reforestación, son mayores los rendimientos cuando se aprovecha la tecnología existente para lograr mayores superficies reforestadas. El caso ilustrado en la figura 6 ha sido una modifición de Holekamp (1958), el autor no ha encontrado maquinaria parecida en América Latina, por lo que sugiere que se haga el intento de adaptar sembradoras mecánicas comunes para la siembra directa. De hecho, según el tamaño de las semillas, es factible introducir germoplasma forestal con una gran variabilidad de semillas comerciales y hasta fertilizantes.

Los vínculos entre la tecnología agrícola y la silvicultura siguen evolucionando. Actualmente, la gama de tecnología desarrollada con base en el tractor agrícola es impresionante. Se podrían citar los casos de China y Finlandia. El caso concreto del tractor chino "Dong Feng" se basa en una máquina diesel de un cilindro con enfriamiento de "chaleco" de agua e impulsado mecánicamente por bandas. Con su configuración básica, el tractor se usa con "rotovator" para hortalizas o en viveros forestales. Desactivando las aspas, se puede usar el motor para impulsar una bomba de agua para riego o para activar otros accesorios como la 


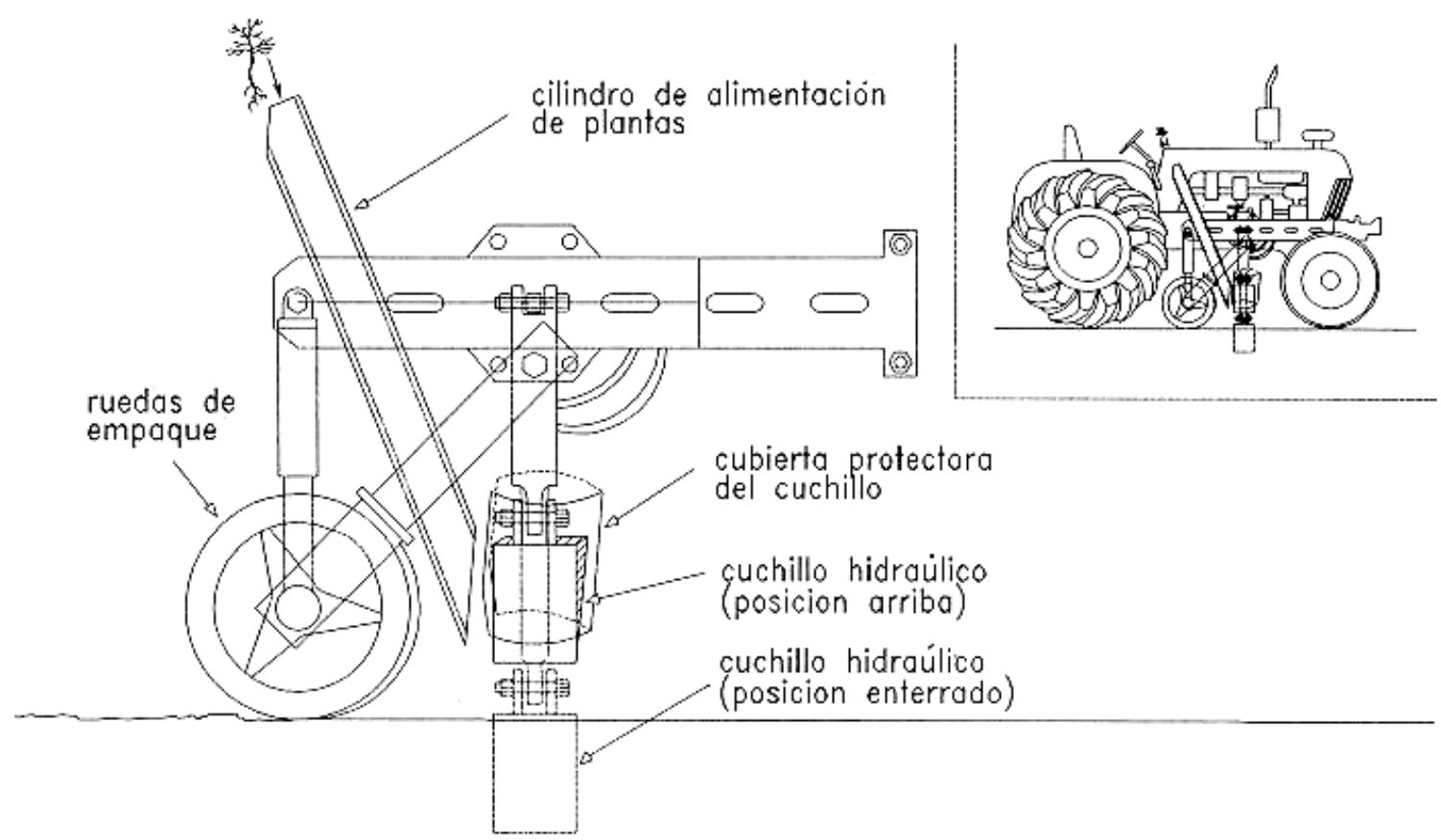

Figura 5. Sistema de inyección de plantas diseñado por el Tecnológico de Luisiana (Altman, 1969)

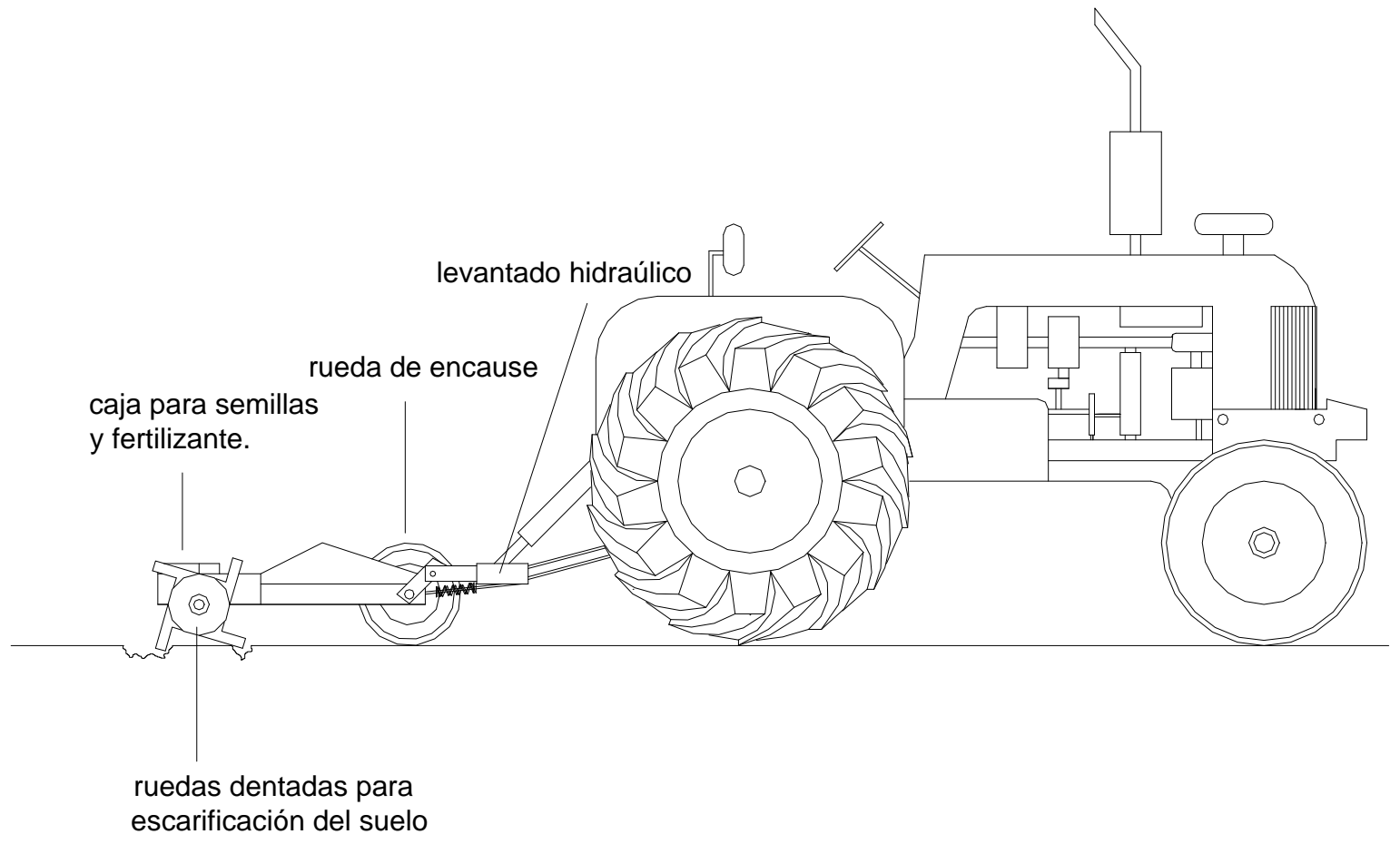

Figura 6. Sembradora de semillas (adaptado de Holekamp, 1958) 
pulidora de arroz. No solamente estos accesorios se pueden usar con la máquina; cuando se engancha un remolque, el motor del Dong Feng tiene capacidad para mover una tonelada de productos a velocidades de hasta 30 kilómetros por hora. Se han utilizado estos tractores para la preparación de camas de trasplante en la producción de plántula a raíz desnuda (Sánchez et al., 1988).

Otro caso de frontera para adquirir maquinaria adaptable a tractores agrícolas para fines forestales se encuentra en los países nórdicos. Hoy en día, entre los países que están incrementando sus ventas de accesorios agrícolas para ampliaciones forestales se encuentra Finlandia. En América Latina se pueden conseguir equipo para desrame y despunte, malacates para arrime, brazos hidráulicos para cargar, astilladoras y los remolques; todos ellos en los modelos básicos para optimizar el trabajo de tractores agrícolas. Por supuesto, no son los únicos casos en donde la tecnología agrícola sirve como base para desarrollar e impulsar la silvicultura, sin embargo, en los países nórdicos un campesino puede ser dueño de tierras arables, así como de "montes" sobre los cuales el potencial agrícola es casi nulo y de no ser tratado con los debidos cuidados se erosiona con gran facilidad. Es decir, se tienen que abordar las mismas alternativas agrícolas, pecuarias o forestales y usar la maquinaria hasta su máxima capacidad y adaptabilidad para cumplir con las tareas. A diferencia de muchos suelos tropicales, las alternativas para los nórdicos han sido claras a través de los siglos. Aprendieron a aprovechar la larga época invernal y la maquinaria agrícola que no se esta usando, para hacer labores silvícolas (sobre todo las relacionadas con corte y transporte) cuando los suelos están congelados y poco erosionables.
El reto de reforestar extensas áreas en los países tropicales, podría superarse con el uso de procesos mecanizados y semimecanizados para las plantaciones forestales (Sánchez et al., 1988). Sin embargo, muchos protagonistas de los programas de reforestación no solamente contemplan la participación del poseedor del bosque, quién recibe remuneración monetaria, sino que no quieren maquinaria argumentando que "deja a su gente sin trabajo". Por ende, se han quedado muy cortos al cubrir las necesidades reales de reforestación, los programas mismos se han vuelto paternalistas e ineficientes por la falta de mecanización, y como consecuencia, con cierta frecuencia reciben menos apoyos a la larga. Entonces, hasta en los casos más extremos de programas cuya meta es el empleo; la tecnología también es importante, sobre todo para minimizar la lentitud y reducir el esfuerzo empleado en hacer las cepas. Por otra parte, el uso de tecnología no solamente puede mejorar el costo-beneficio de los proyectos, sino interesar al usuario a cualquier nivel socioeconómico. Para abrir el surco tras el paso de maquinaria agrícola se logra minimizar la parte más laboriosa y desalentadora de la plantación.

En el trópico, la maquinaria agrícola es ampliamente utilizada; tractores con llantas neumáticas sencillas, dobles y con canastas son complementados con tractores de oruga en las pendientes. Los usos más frecuentes del suelo incluyen los cultivos de caña de azúcar y de arroz y la ganadería. La mecanización de las actividades agrícolas en el otoño y primavera es casi reglamentaria en las labores de barbecho, siembra, labores de cultivo y cosecha. El chapeo de potreros también es común con la devanadora arrastrada por tractores. Es frecuente, sobre todo en los programas oficiales, que la maquinaria esté sin uso durante meses. 
Los forestales tendrán que acercarse más para conocer sobre la disponibilidad de maquinaria y así promover una utilización más completa y amplia en las plantaciones forestales ya sean con fines comerciales o de reforestación.

No siempre se puede costear un tractor de oruga. Como medida práctica es recomendable usar el mínimo de maquinaria, sobre todo cuando se trata de pendientes fuertes, suelos erosionables 0 cuando su costo de alquiler se tiene que pagar con alguna tasa de interés alta. Existen, desde luego, en los ambientes arroceros, canastillas para cubrir las llantas e inclusive la práctica de usar doble rodada cuando los campos están inundados. En el caso de la vegetación secundaria, puede resultar peor en el campo inundado y resbaloso dado que la vegetación no permite que avance del tractor convencional. Si se realizan adaptaciones a un tractor convencional, este puede entrar en un bosque secundario espeso si cuenta con personal de apoyo para abrir brechas manualmente, siempre y cuando no se atasque; de lo contrario, puede ser peligroso el uso de maquinaria liviana en zonas de vegetación densa sin una caseta de protección para el operador.

En países en vías de desarrollo se encuentra maquinaria tanto liviana como pesada para la agricultura o para la construcción. La maquinaria está en manos de consorcios poderosos que han propiciado la neofrontera agropecuaria. En estos casos, por falta de suficientes conocimientos y herramientas básicas, los forestales han perdido oportunidades excelentes para avanzar en la tecnificación de la reforestación como proyecto de interés comunitario.

La gran distancia a que se encuentra la ciencia silvícola respecto al desarrollo y a la práctica de las actividades de frontera, sin duda, impide el avance de la reforestación en América Latina. En la planeación se deben contemplar las plantaciones tecnificadas. Claro está que la preparación de los terrenos para las plantaciones forestales puede hacerse a mano o con maquinaria; pero para la preparación mecánica se requiere maquinaria. Para poner en operación esta maquinaria habrá que calcular los costos de mantenimiento, arrendamiento o amortización. De no tenerla disponible, se incurre en costos que los programas de pequeña o mediana escala difícilmente podrían alcanzar. Estos costos pueden estar mejor repartidos, por el uso de la maquinaria en otras actividades sobre todo de beneficio social (y no necesariamente forestal) o junto con otras obras que haga la empresa, la finca o el programa. Adicionalmente se necesitan combustibles, refacciones y el entrenamiento del personal. El acercamiento entre los forestales con los demás programas -particulares y del gobierno- abre posibilidades para que la reforestación sea mas eficiente y a escalas mayores.

No obstante, el costo inicial de capital no es un obstáculo grave cuando se renta maquinaria por horas o cuando la plantación forma parte de un programa gubernamental. De cualquier manera, el uso de maquinaria ligera es preferible porque reduce los costos. En general, las operaciones de preparación y limpieza mecánica son más baratas que las manuales, pero la elección entre uno y otro métodos debe ser analizada caso por caso según las particularidades de cada lugar (Endean y Hoecking, 1973). La maquinaria que se utiliza depende en primer lugar de la que haya disponible. Las características del sitio tales como la pedregosidad del suelo y la densidad del matorral o la pendiente, también son determinantes para la 
selección de maquinaria (McKenzie et al., 1984; Noecker, 1967).

En países donde es común el uso de maquinaria trasplantadora se suelen utilizar tractores que arrastran y al mismo tiempo derriban la vegetación arbustiva, no requiriéndose de una esmerada preparación del sitio. Cuando el terreno es adecuado, la plantación mecanizada o semimecanizada resulta muy superior en rendimiento, comparada con aquellas establecidas por métodos manuales, la primera alcanza un rendimiento mínimo de entre 500 y 1500 plántulas sembradas por hora (McKenzie et al., 1984). De acuerdo con los autores citados, los costos de la maquinaria están incrementándose y para que la operación sea redituable, una trasplantadora de árboles tiene que alcanzar un mínimo de 540 a 600 árboles por hora con un costo inferior a $\$ 0.12$ dólares (75 centavos mexicanos) por cada planta que se trasplante con sistema mecanizado. Para algunos, esta velocidad parece inalcanzable. Sin embargo, Troup (1952) reportó rendimiento de 4,000 a 5,000 plántulas trasplantadas por día con la tecnología rudimentario de su época.

Finalmente, la tecnología no tiene que ser factor decisivo para que la reforestación sea exitosa. Lamentablemente, en épocas recientes los proyectos de reforestación con mayores apoyos financieros están en manos de políticos y no de técnicos que bien pudieran participar en la planeación y ejecución. Para Laarman (1995) es casi incomprensible que los esfuerzos para hacer las leyes, la política forestal y la promoción sobre las metas de reforestación absorban casi todo el tiempo y la energía de las agencias forestales en el tercer mundo. Desde su punto de vista, más del $90 \%$ de la reforestación de los países más pobres es llevado a cabo por medio de los organismos no gubernamentales.

La discusión sobre sustentabilidad ha llegado a vislumbrarse, en muchos ámbitos, como el balance entre lo reforestado y la deforestación. Toman (1992) considera que para entender la sustentabilidad se debe partir de dos conceptos implícitos: por una parte, debe existir la responsabilidad de manejar el recurso para generar capital social, de tal forma que las actividades actuales sean equitativas y eviten la degradación de los recursos para beneficio de generaciones futuras y por otra parte, se debe especificar qué es lo que se pretende sostener. En ninguna época de la humanidad se ha discutido tanto como ahora respecto a las actividades del campo que pueden provocar la pérdida de diversidad biológica, calentamiento global, deforestación, degradación ambiental entre otros efectos indeseables. Por otro lado, ahora está más claro que las mismas actividades pueden darse en forma moderada, y de hecho, siempre han tenido el respaldo de grupos cuando existen avances en la organización social, así como conocimientos y técnicas que generan el bienestar humano a perpetuidad. Este último punto de vista no es aceptable por ecologistas pero sí por algunos economistas.

\section{CONCLUSIONES}

Existe la creencia de que en la actualidad los daños al medio ambiente por la contaminación y deforestación no tiene paralelos en la antigüedad. Se puede afirmar que ésta es la época de máxima destrucción de los recursos naturales y de la expansión tecnológica, tanto por el número de habitantes en el planeta como por los motivos ya bien documentados. No obstante lo anterior, algunos apoyos y/o soluciones, están a la mano. La tecnología 
misma no ha sido suficientemente explotada para apoyar la tarea de rehabilitar las áreas, ni siquiera cuando se desarrolló la maquinaria trasplantadora para superar retos de reforestación similares a los que actualmente se presentan en los países latinoamericanos.

Los acontecimientos de la "modernización del campo" actual en América Latina, en donde la tecnología ha llegado a las manos de la gente del campo a ritmos acelerados, son de alguna manera semejantes a la frontera angloamericana del pasado. Las plantaciones mecanizadas y semimecanizadas no constituyen ninguna panacea. Sin embargo, si pueden resolver muchas dificultades siempre y cuando la escala de operación y capital lo permitan y este ligada con la tecnología disponible. El uso eficiente de recursos tecnológicos y de material vegetal por recursos humanos entrenados, será de los pocos mecanismos disponibles para atender las extensiones tan urgidas de reforestación. Para enfrentar el reto de la reforestación en el trópico se requerirá que los forestales entiendan a la deforestación bajo un trasfondo cultural historico y como han sido realizados los esfuerzos por resolverla. Los ejemplos presentados tienen elementos culturales muy distintos que deben ser analizados con detenimiento, incluyendo la clara preferencia hacia el uso de especies nativas en la reforestación mecanizada. En el caso de EUA la colonización de tierras arrojó recursos para cubrir los costos fiscales y ecológicos para la reforestación. Este caso contrasta con el reparto de las tierras en América Latina con la ganaderización que, frecuentemente se ha financiado con préstamos externos y cuyos pagos son tarea de todos los habitantes del país afectado y no solo del sector beneficiado.

\section{AGRADECIMIENTOS}

El presente trabajo no habría sido posible sin el esfuerzo y dedicación del D.I. Enrique Martínez-Pinillos C., a quien se deben la confección de las figuras y de la M. en C. Ma. Teresa Pulido quien se ha dedicado a desenredar el texto presente y ha aportado valiosas observaciones sobre aspectos culturales.

Este trabajo fue realizado con fondos fiscales asignados al Departamento de Productos Forestales y Conservación de Bosques del Instituto de Ecología, A.C. con la clave 903-13.

\section{REFERENCIAS}

Altman, J.A. 1969. LA. Tech's hydraulic tree planter. American Pulpwood Association. Tec. Rel. 69-R-68:1-3.

Anduaga M., J. 1988. Respuesta de maculis (Tabebuia rosea) y siricote (Cordia dodecandra) a siembra mecanizada y manual bajo dos condiciones ecológicas: dosel protector y campo abierto, en la Chontalpa-Tabasco. Tesis de Maestría en Ciencias. Col. de Postgr. Montecillos, México. 162 pp.

Bagley, W.T. 1973. Heavy duty tree planting machine developed in Nebraska. Tree Planters' Notes 2:27-28.

Bamford, J. y J. Bamford 1981. Horse loggers. In: R. Raphael (Ed.) Trees talk; the people and politics of timber. Island Press, Covelo, Ca.:132-144.

FAO. 1983. Tecnología básica en operaciones forestales. Estudio FAO: montes. Organización de las Naciones Unidas para la Agricultura y la Alimentación. Roma. 122 p. 
Hamilton, L. S. 1990. Restoring of degraded tropical forests. p:113-122.

Hensel, J.A. 1958. Rootspread lake states model tree planter. American Pulpwood Association. Tec. Rel. No. 58-R29: 1-2.

Hensel, J.A. 1959a. The "conservator" tree planter. American Pulpwood Association. Tec. Rel. No. 59-R16:1-2.

Hensel, J.A. 1959b. The "goodmonson" tree planter. American Pulpwood Association. Tec. Rel. No. 59-R17:1-4.

Hensel, J.A. 1959c. The "forester" tree planter. American Pulpwood Association. Tec. Rel. No. 59-R3:1-4.

Hensel, J.A. 1961. "A homemade tree planter". American Pulpwood Association. Tec Rel. No. 61-R35:1-3.

Hill, J. H. 1964. Underplanting with Beloit (Lowther) tree planters in East Tennessee. American Pulpwood Association. Tec. Rel. 64-R-56:1-4.

Holekamp, J.A. 1958. Concord furrow seeder. American Pulpwood Association Tec. Rel. No. 58-R-31:1-4.

Honadle, G. 1992. Institutional constraints on sustainable resource use: lessons from the tropics showing that resource overexplotation is not just an attitude problem and conservation education is not enough. p:90-96. In: Aplet, G.H., Olson, J.T., y V.A. Sample (Eds.) Defining sustainable forestry. Island Press, Covelo, $\mathrm{Ca}$.
Laarman, J. 1995. The political economy of forestry. Sylvanet Volume 7 issue 3. Sin paginación.

Lawyer, J.N. 1974. Review of the literature on mechanized planting systems. Chapter 4. Analyis of mechanized systems for planting trees for reforestation. Dept. of Agricultural Engineering. University of California. Davis, Ca.:29-38.

McKenzie, D.W., D.C. Hotfield y K.K. Kyman. 1984. Tree-planting machines, Can you afford one?. Tree Planters' Notes 2:8-11.

McKnight, J.R. 1976. Bradlley spot planter. American Pulpwood Association. Tec. Rel. No. 76-R-46:1-4.

Noecker, N. 1967. A critical analysis of machine planting. American Christmas Tree Journal 11(1):5-6.

Pimentel B., L. 1995. Comunicación personal.

Sánchez A., M. Keyes H., A. Trinidad S. y V. Cetina V. 1988. Comparación de tres técnicas de producción en vivero de 15 especies de pino. Agrociencia No. 72:926.

Schule, W. 1990. Landscapes and climate in prehistory: Interactions of wildlife, man and fire. In: J. G. Goldammer, Ed. Fire in the tropical biota. Ecological Studies 84. Ecosystem processes and global challenges. Springer-Verlag. Berlin. p: 273-318.

Shirley, H. L. 1952. Forestry and its career oportunities. McGraw-Hill Book Company. 492 pp. 
Steinbeck, K. y R.G: McAlpine. 1973. Furrow planting of American sycamore cuttings. Tree Planters' Notes 4:22-24.

Toman, M.A. 1992. Defining an economics of sustainable forestry: General concepts. In: G.H. Aplet, J.T. Olson y V.A. Sample (Eds.). Defining sustainable forestry. Island Press. Covelo, Ca. p: 270-279.
Troup, R.S. 1952. Silvicultural systems. 2nd ed. Ed. by E.W. Jones. Oxford Univ. Press. London. 199 pp.

US Forest Service. 1967. List of commercial tree planters. Tree planters' Notes 18(1). 13 pp.

WRI. 1990. World Resources. 1990-91. A report by the World Resources Institute. Oxford University Press. $383 \mathrm{pp}$. 